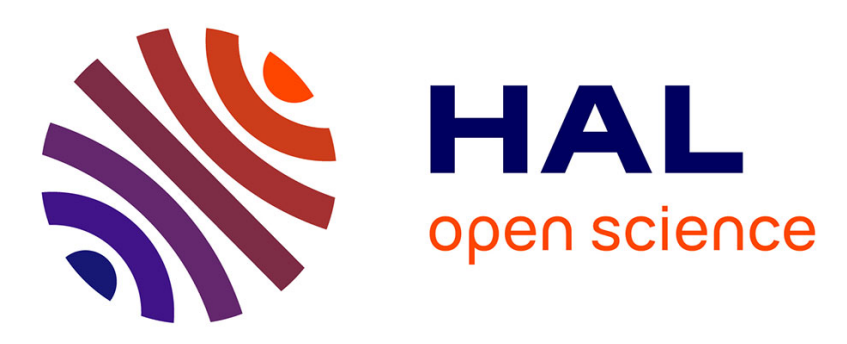

\title{
Slow pulses in disordered photonic-crystal waveguides
} Alexandre Baron, Simon Mazoyer, Jean-Paul Hugonin, Philippe Lalanne, Andrea Melloni

\section{To cite this version:}

Alexandre Baron, Simon Mazoyer, Jean-Paul Hugonin, Philippe Lalanne, Andrea Melloni. Slow pulses in disordered photonic-crystal waveguides. Applied optics, 2011, 50 (31), pp.G113. 10.1364/AO.50.00G113 . hal-00675340

\section{HAL Id: hal-00675340 \\ https://hal.science/hal-00675340}

Submitted on 29 Feb 2012

HAL is a multi-disciplinary open access archive for the deposit and dissemination of scientific research documents, whether they are published or not. The documents may come from teaching and research institutions in France or abroad, or from public or private research centers.
L'archive ouverte pluridisciplinaire HAL, est destinée au dépôt et à la diffusion de documents scientifiques de niveau recherche, publiés ou non, émanant des établissements d'enseignement et de recherche français ou étrangers, des laboratoires publics ou privés. 


\title{
Slow pulses in disordered photonic-crystal waveguides
}

\author{
Simon Mazoyer, ${ }^{1}$ Alexandre Baron, ${ }^{1}$ Jean-Paul Hugonin, ${ }^{1}$ \\ Philippe Lalanne, ${ }^{1,2, *}$ and Andrea Melloni ${ }^{3}$ \\ 'Laboratoire Charles Fabry de l'Institut d'Optique, CNRS, Univ Paris-Sud, \\ Campus Polytechnique, RD 128, 91127 Palaiseau Cedex, France \\ ${ }^{2}$ Laboratoire Photonique, Numérique et Nanosciences, CNRS, Univ Bordeaux 1, \\ Institut d'Optique, 33405 Talence cedex, France \\ ${ }^{3}$ Politecnico di Milano, Dipartimento di Elettronica e Informazione, I-20133 Milan, Italy \\ *Corresponding author: philippe.lalanne@ institutoptique.fr
}

Received 1 July 2011; revised 7 September 2011; accepted 30 September 2011; posted 4 October 2011 (Doc. ID 150193); published 25 October 2011

\begin{abstract}
Using a 3D fully-vectorial coupled Bloch-mode method, we present a systematic study of the transport of slow-light pulses in single-mode photonic-crystal waveguides $(\mathrm{PhCW})$ with a realistic disorder model. For the intermediate regime corresponding to waveguide lengths of the order of the mean-free path $(3 \mathrm{~dB}$ attenuation), we show that the group-velocity has a strong impact on the pulse broadening and distortion, limiting the practical use of PhCW to group indices below $\approx 50$. For smaller group velocities, the pulse experiences an additional delay and the group-velocity is no longer a meaningful quantity. (C) 2011 Optical Society of America
\end{abstract}

OCIS codes: $\quad 130.5296,030.5770,290.4210,320.5550$.

\section{Introduction}

Photonic-crystal $(\mathrm{PhC})$ structures offer a rich degree of control over light-matter interactions, leading to trapped and slow-light modes buried within a forbidden photonic band gap. In the more practical planar $\mathrm{PhC}$ semiconductor systems, waveguide modes can be completely bound below the light line and thus can be theoretically lossless. However, manufacturing imperfections result in fabrication disorder that break the translational invariance of a nominally perfect lattice and cause scattering of the bound modes. Despite great initial premises [1-3] , disorder is presently believed to be a severe limitation for the deployment of slow-light $\mathrm{PhC}$ waveguides (PhCW) For instance, recent studies have shown that the waveguide attenuation quadratically increases with the group index [4-6], that the transmission prob-

0003-6935/11/31G113-05\$15.00/0

(C) 2011 Optical Society of America ability distribution rapidly broadens as the groupvelocity decreases even for modest waveguide lengths of a few tens of wavelengths [7]. In addition, in agreement with earlier works [8], direct measurements of the dispersion curve in $k$-space [9] have also shown that, for group velocities $v_{g}<c / 25$, the groupvelocity concept loses its relevance. Finally, it has been predicted that the light transport is no longer dominated by a single propagative Bloch-mode for slow-light, but by a complex standing-wave superposition formed only by the fundamental backward and forward Bloch-modes. These modes are Fabry-Perot extended resonances assisted by a series of more localized quasi-states, similar to coupled cavity states $[7,10]$ at a given wavelength, which are remnant of light localization in these waveguides, as has been observed for intentionally large disorders $[11,12]$.

Although for on-chip optical processing applications the information is encoded on high repetitionrate pulses, slow-light transport in $\mathrm{PhCW}$ has been mainly studied in the frequency-domain up to now, 
either theoretically with frequency methods or experimentally with tunable lasers to our knowledge. Exceptions however arise for recent studies on delay lines [13] and on various nonlinearities of slow-light pulse propagation in $\mathrm{PhCW}$ [14-16]. However, even in these studies, fabrication imperfections are assumed to impact only the effective linear attenuation. This assumption is made theoretically in the so-called nonlinear Schrödinger equation [14] and is used to exploit experimental data, but no systematic discussion is ever provided on any eventual pulse distorsion.

In contrast, the present work provides a direct study of the impact of fabrication imperfections on the propagation of slow-light pulses in PhCWs. It is not devoted to the extreme regime of pulse localization, but rather to intermediate regimes that are relevant for practical situations. Hereafter, referring to the terminology classically used for the electronic transport in 1D systems [17], we distinguish the ballistic regime that is obtained for waveguide lengths $L$ much smaller than the mean-free-path $\ell$ (defined as the waveguide length corresponding to a $3 \mathrm{~dB}$ attenuation) and the localization regime obtained for $L \gg \ell$. More specifically, we will be concerned by the intermediate regime, $L \approx \ell$, which offers a good compromise between loss and delays. Section $\underline{2}$ presents the $\mathrm{PhC}$ waveguide under study, the disorder model and the numerical method used to calculate pulse propagation and to collect statistics on ensemble average. In Section $\underline{3}$, the broadening, damping, and dispersion of picosecond pulses is analyzed for several group velocities, keeping the waveguide lengths of the order of the mean-free path. Section $\underline{4}$ offers a summary of the main results.

\section{Disorder Model and Numerical Method}

Hereafter, we consider a $\mathrm{PhC}$ waveguide obtained by removing a single row of holes in the $\Gamma \mathrm{K}$ direction of a hexagonal lattice of air holes in a $220 \mathrm{~nm}$-thick silicon layer (refractive index 3.45), see Fig. 1(a). For a lattice constant $a=420 \mathrm{~nm}$ and a hole radius
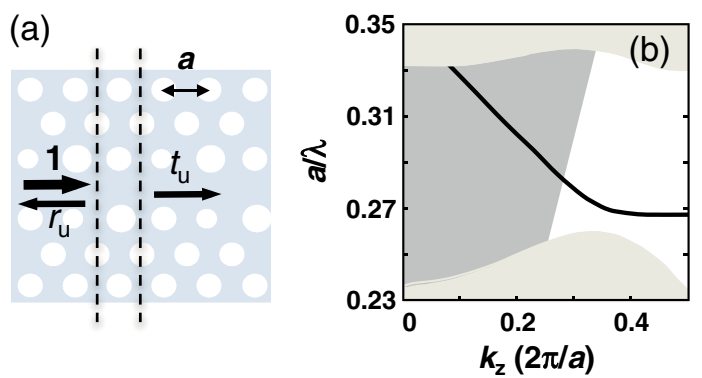

Fig. 1. (Color online) Photonic-crystal waveguide considered in the present work. (a) The disorder model assumes that only the hole-size of the two inner-rows is varying with a Gaussian-random distribution of standard deviation $\sigma$. (b) Dispersion diagram of the TE-like Bloch-mode of the ideal waveguide, calculated with a $3 \mathrm{D}$ fully-vectorial method for a $220 \mathrm{~nm}$ thick silicon layer (refractive index 3.45), for a lattice constant $a=420 \mathrm{~nm}$ and a hole radius $r=0.3 a$. $r=0.3 a$, the PhC waveguide supports a single propagating TE-like Bloch-mode with a group-velocity that gradually decreases as the wavelength increases up to the cutoff wavelength $\lambda_{c} \approx 1560 \mathrm{~nm}$ at the boundary of the first-Brillouin zone $(a / \lambda \approx 0.27)$. The dispersion curve, calculated as $d \omega / d k_{z}$ with a $3 \mathrm{D}$ fully-vectorial modal method [18], is shown with the black curve in Fig. 1(b).

Hereafter, we use a hole-size disorder model, in which we assume that the hole radii of the two inner rows, see Fig. 1(a), are randomly varied around their mean nominal value of $r=0.3 a$ with a statistical Gaussian distribution of standard deviation $\sigma=$ $1.7 \mathrm{~nm}$. We further assume a short-range disorder, so that two distinct etched holes have fully-independent deformations. We neglect any potential vertical asymmetry of the hole. Indeed, the model is likely to be simplistic. However, we note that the two inner rows are dominantly interacting with the waveguide mode field; it is only for very small group velocities $\left(n_{g}>100\right.$ not considered hereafter) that the Blochmode spreads into the $\mathrm{PhC}$ cladding and significantly interacts with the second-inner rows. In addition, we note that, as shown in [19], with a standard deviation $\sigma=1.7 \mathrm{~nm}$, the model quantitatively predicts many salient features of the slow-light transport in $\mathrm{PhCW}$ obtained with state-of-the-art nanofabrication facilities.

The computation of pulse propagation in disordered $\mathrm{PhCW}$ is challenging, because one is concerned by very small perturbations in the nanometer range, with long propagation distances of the order of a few hundreds of micrometers and with high refractive index contrast. The coupled Bloch-mode method that we use hereafter is highly accurate. It has been elaborated to overcome the limitations of earlier approaches based on perturbation theory [17] and as shown by comparison with experimental data [7,19], it is able to quantitatively predict many important features of slow-light transport in $\mathrm{PhCW}$. In brief, the method relies on a two-step procedure. The first step consists in calculating the elementary singlecell scattering coefficients $\left[r_{u}\right.$ and $t_{u}$ in Fig. 1(a)] for every possible unit-cell disorder instance labeled by $u$. This computation is central in the analysis and is performed with a fully-vectorial Fourier-modal method that allows satisfying ingoing and outgoing wave conditions in periodic media [18]. At this level, there is no approximation, other than the inevitable Fourier-space sampling that is finite due to memory requirements, and $r_{u}$ and $t_{u}$ are calculated with a high accuracy. The second step consists in calculating the transmittance $T_{L}(\omega)=\left|t_{L}(\omega)\right|^{2}$ (and the reflectance $R_{L}$ ) of a perturbed waveguide-section with a finite length $L$. For this, it is assumed that solely the fundamental TE-like Bloch-mode participates in the in-plane energy transport at frequency $\omega$. Thus, it is sufficient to consider $2 \times 2$ scattering matrices, $s_{u}=\left(\begin{array}{ll}t_{u} & r_{u} \\ r_{u} & t_{u}\end{array}\right)$, to model the transport properties and $R$ and $T$ can be easily computed using classical 
$2 \times 2$ scattering-matrix products [20]. Because of the reduction in matrix sizes, the computational loads are drastically reduced and statistics are straightforwardly collected, even for long waveguide sections.

It is important to understand the approximations made with the single-mode frequency-domain approach. First, we note that since $\boldsymbol{s}_{u}$ is calculated using a fully-vectorial approach, the scattering (including out-of-plane losses) of every elementary event is rigorously handled at every frequency. Additionally, in-plane multiple scattering is inherently taken into consideration by $2 \times 2$ scattering-matrix products. The sole approximation stems from the fact that one disregards the potential recycling of photons that are scattered out in the cladding by the scatterers. Actually, before fully leaking out of the waveguide, these photons may be scattered by another nearby scatterer and a fraction of them may be recycled back into the waveguide. The interested reader may find a discussion of the impact (generally weak for moderate $n_{g}$ 's) of the multiple scattering of radiated photons in the supplementary-information document in [17]. All the previous calculations are performed in the frequency-domain, they are repeated for many frequencies, and finally, the timedependent pulses are obtained by classical Fourier decomposition techniques.

\section{Pulse Propagation: Computational Results}

Let us start by considering the transmission spectra calculated with the coupled Bloch-mode method. Figure 2 shows three spectra obtained for three waveguides with lengths, $L=100 a, 200 a$, and $500 a$ for $\sigma=1.7 \mathrm{~nm}$. In the absence of any disorder, $T_{L}(\lambda)$ should be equal to 1 , since the TE-like Bloch-mode is truly guided in the spectral range of interest. The main feature of the spectra is their oscillating behavior reinforced by spikes for large $n_{g}$ 's. We note that the oscillation frequency increases and that the spikes become narrower as the waveguide length increases. The spikes have been recently observed experimentally and have been interpreted as Fabry-Perot extended resonances assisted by a series of localized states [7].

Next we consider the propagation of Gaussian pulses, with an amplitude denoted by $s_{0}(t)=$ $B \exp \left[-t^{2} / 2 \tau^{2}\right]$. Hereafter, we consider a pulse duration of $\tau=7.5 \mathrm{ps}$, corresponding to a $25 \mathrm{ps}$ fullwidth-half-maximum temporal width, but similar qualitative conclusions are also obtained with slightly shorter pulses. The duration of $7.5 \mathrm{ps}$ corresponds to a spectral width of $0.1 \mathrm{~nm}$ that is represented by the colored area in Fig. $\underline{2}$. Figure $\underline{3}$ summarizes the main useful results obtained for modest waveguide lengths $L=100,200$, and $500 a$ and for three group indices, $n_{g}=20,50$, and 70 . For the following discussion, it is interesting to keep in mind the corresponding mean-free-paths, equal to $\ell \approx 10^{4} a, 200 a$ and $40 a$, respectively. For every case, we consider 100 instances of independent disorder realizations and we consider the ensemble average of the natural logarithm of the waveguide transmis$\operatorname{sion}\langle\ln (T)\rangle$ as a function of $L$. This quantity is a linearly decreasing function of $L$ and its slope provides the mean-free path $\ell$ by $\langle\ln (T)\rangle=-L / \ell$ [21]. Then, for the 100 instances, we calculate the transmitted pulses for an incident pulse with a unit energy, $\int\left|s_{0}(t)\right|^{2} \mathrm{~d} t=1$. Only five typical pulses are shown in the figure for the sake of clarity. The 100 samples allow us to compute ensemble-averaged physical quantities, such as the mean pulse energy $I=$ $\int|s(t)|^{2} \mathrm{~d} t$ or the mean effective energy-propagation delay $[\underline{22}, \underline{23}]$

$$
\Delta t_{E}=I^{-1} \int t|s(t)|^{2} \mathrm{~d} t
$$

where $s(t)$ is the amplitude at time $t$ of the pulse transmitted by a $\mathrm{PhC}$ waveguide of length $L$. Note that $\Delta t_{E}$ is directly related to the energy velocity $v_{E}$ by the relationship $v_{E} \Delta t_{E}=L$ [23].

It should be noted that the group-velocity dispersion (GVD) is taken into account in the previous calculations. As evidenced by the black-dashed curves in Fig. 3, which represent pulses transmitted through the disorder-free waveguides, the GVD plays no role in the pulse broadening. This is consistent with the fact that the waveguide lengths $L$ considered in the study are much smaller than the dispersion lengths $L_{D}=\left|\beta_{2}\right| / \tau^{2}$ ( $\beta_{2}$ being the second-order dispersion coefficient), i.e. the propagation distances for which the pulse durations are doubled. The latter are $L_{D} \approx 80,000 a, 10,000 a$, and $5400 a$ for $n_{g}=20$, 50 , and 70 , respectively.

Let us first consider the transmitted pulses for $n_{g}=20$ (left column in Fig. 3 ). In this case, the three waveguide lengths that are considered are much smaller than the mean-free-path $\left(\ell \approx 10^{4} a\right)$ and the
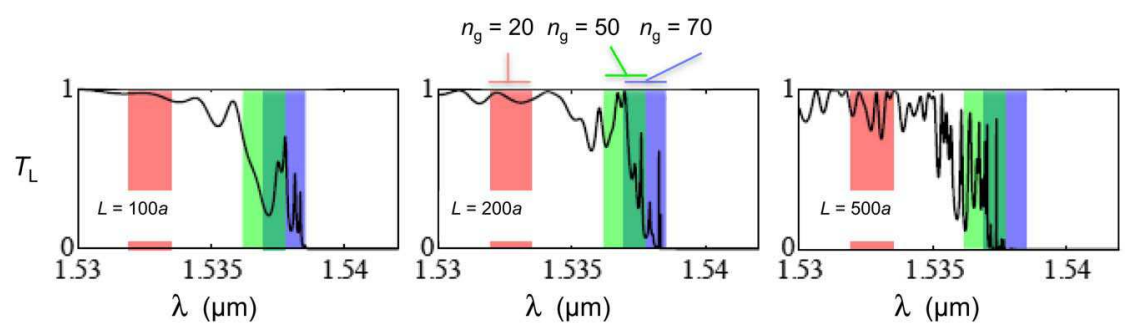

Fig. 2. (Color online) Examples of calculated transmission spectra for three waveguide lengths $L=100,200$, and 500a. The pink, lightgreen, and blue spectral intervals that are respectively centered at $n_{g}=20,50$, and 70 , are $0.2 \mathrm{~nm}$ large. This corresponds to spectral intervals twice larger than the 25 ps pulse FWHM. 

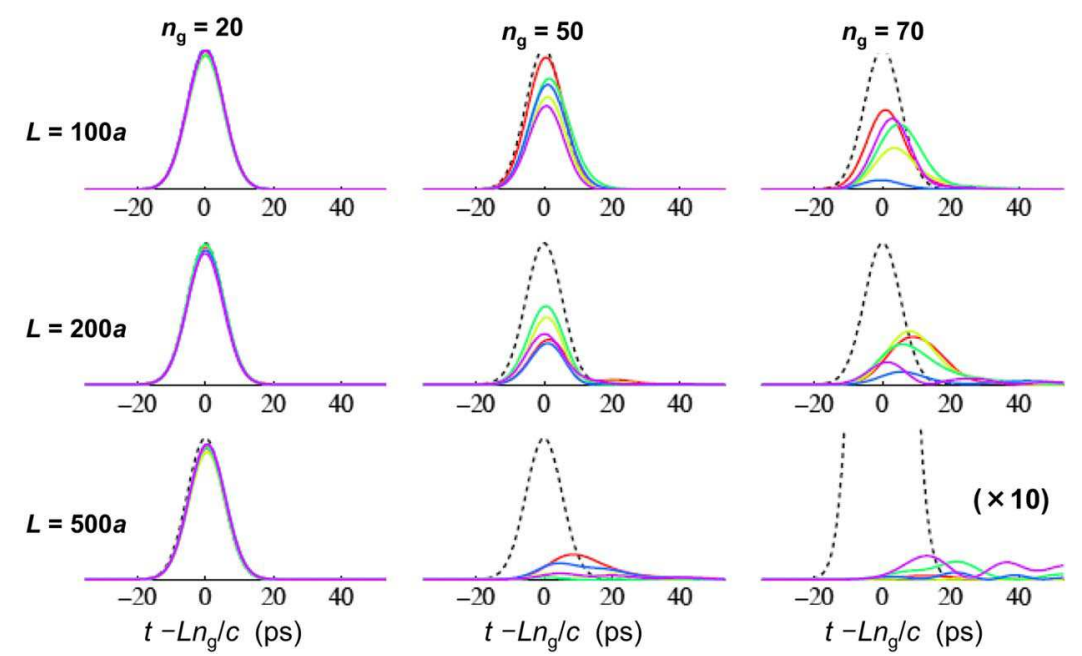

Fig. 3. (Color online) Pulse distorsion after propagation through a finite-length disordered PhC waveguide. The colored curves correspond to typical examples obtained for different disorder realizations, calculated for $\sigma=1.7 \mathrm{~nm}$. For comparison, the black-dashed curves correspond to the ideal case, in which the same pulse propagates into the PhC waveguide in the absence of disorder. Three lengths, $L=100,200$, and $500 a$, and three group indices, $n_{g}=20,50$, and 70 are considered.

transport is essentially ballistic: the pulses are only weakly distorted as evidenced by comparison with the black-dashed curves. The group-velocity is then equal or close to the energy velocity as can be seen in Table 1, which additionally shows the averaged transmitted-pulse energy $\langle I\rangle$.

For $n_{g}=50$ (center-column), the results are different; the transmitted pulses are significantly attenuated and broadened. They remain Gaussian-like for $L=100 a$, but for $L=200 a$ and $500 a$, a tail is obtained for a few pulses and the pulse shapes are no longer Gaussian-like. We additionally note that, because of the imperfections, the pulses experience an additional delay $\left(\Delta t_{E}-L n_{g} / c\right)$ that is easily noticeable for the transmitted pulses obtained for $L=$ $500 a$, since it varies between 5 and $10 \mathrm{ps}$ from one pulse to another. As it corresponds to the meanfree-path for $n_{g}=50$, the waveguide length $L=$ $200 a$ (central plot) is especially interesting (see Table 1). In this case, the normalized averaged pulse energy $\langle I\rangle$, calculated over the 100 independent disorder realizations, is 0.52 . However, although the pulse attenuation corresponds to an acceptable $\sim 3 \mathrm{~dB}$ loss, the transmitted-pulse energies strongly vary from one disorder realization to another. These statistical fluctuations can be seen in the central window of Fig. 3. The intensity standard deviation is $\sigma_{I}=\left\langle(I-\langle I\rangle)^{2}\right\rangle=0.19$, a rather large value that

Table 1. Statistics for the Pulses Displayed in Fig. $\underline{3}^{a}$

\begin{tabular}{l|lll|lll|lll}
\hline \multicolumn{1}{c|}{$L$} & \multicolumn{3}{c|}{$100 a$} & \multicolumn{3}{c|}{$200 a$} & \multicolumn{3}{c}{$500 a$} \\
\hline$n_{g}$ & 20 & 50 & 70 & 20 & 50 & 70 & 20 & 50 & 70 \\
$\langle I\rangle$ & 0.97 & 0.70 & 0.44 & 0.95 & 0.52 & 0.23 & 0.88 & 0.25 & 0.05 \\
$\sigma_{I}$ & 0.02 & 0.18 & 0.20 & 0.05 & 0.19 & 0.14 & 0.07 & 0.13 & 0.05 \\
$\Delta t(\mathrm{ps})$ & 2.8 & 7 & 10 & 5.6 & 14 & 20 & 14 & 35 & 50 \\
$\left\langle\Delta t_{E}\right\rangle(\mathrm{ps})$ & 2.8 & 8.3 & 14 & 5.7 & 18 & 40 & 14.5 & 61 & 165 \\
\hline
\end{tabular}

${ }^{a}\langle l\rangle$ represents the averaged transmitted-pulse energy. $\Delta t=$ $L n_{g} / C$ is the theoretical group delay. $\Delta t_{E}$ is the effective energy delay defined by Eq. (1). precludes reproducible characteristics from one waveguide to another. In addition, we note that the averaged effective delay defined in Eq. (1) is $18 \mathrm{ps}$, a value that is $4 \mathrm{ps}$ larger than the theoretical delay for a perfect waveguide $L n_{g} / c=14 \mathrm{ps}$. As a matter of fact, the energy velocity is $30 \%$ smaller than the group-velocity.

The situation is even worse for $n_{g}=70(\ell \approx 40 a)$. For the same waveguide length $L=200 a,\langle I\rangle=0.23$, $\sigma_{I}=0.6$ and the averaged energy velocity is twice smaller than the group-velocity $\left(\left\langle\Delta t_{E}\right\rangle=40 \mathrm{ps} \approx\right.$ $\left.2 L n_{g} / c\right)$. In the pure localization regime obtained for instance for $n_{g}=70$ and $L=500 a(L \approx 10 \ell)$ (left column in Table 1), pulses are highly damped and distorted $\left(\left\langle\Delta t_{E}\right\rangle=3 L n_{g} / c\right.$ and $\left.\sigma_{I} \sim\langle I\rangle\right)$. The pulses are so distorted that the delay between the leading and trailing edges of the transmitted pulses is much larger than the mean energy-propagation delay $\left\langle\Delta t_{E}\right\rangle$. Therefore, neither the group-velocity nor the energy velocity are meaningful quantities.

\section{Conclusion}

In summary, we have studied the propagation of pulses in PhCW in the presence of realistic disorder. In the ballistic regime, pulses are transported virtually without any deformation. The interesting regime occurs for waveguide lengths approximately equal to the mean-free-path. For this intermediate regime, the pulse attenuation is reasonably weak, $\sim 3 \mathrm{~dB}$ on average, but interestingly, we find that for slow-down factors higher than ten, the sharp and intense peaks observed in the spectral domain (Fig. 1) are partly smoothed in the temporal domain (Fig. 2 ), due to filtering by the spectral window associated to the pulse width. Thus the transmitted-pulse shapes remain Gaussian-like and the energy velocity, not the group-velocity, is still a relevant quantity to consider. It is only for larger slow-down factors or longer waveguide lengths that the pulse distortion is so large that both energy and group velocities are no 
longer meaningful quantities. The same type of calculations as those carried out in this study could be performed for dispersion-engineered waveguides to show that pulse distortion would still occur. However, we believe that, in principle at least, the distortion will remain in these type of structures and will not significantly change the group-velocity boundaries for operation.

As evidenced by our results, we believe that pulse broadening and distortion in $\mathrm{PhCW}$ is due to disorder and not to GVD of the ideal waveguide. As evidenced by all black-dashed pulse shapes in Fig. 3, the GVD negligibly impacts the pulse shape for waveguide lengths equal to the mean-free-path. In addition, we believe that a better knowledge of the pulse distortion is essential for further design of slow-light $\mathrm{PhC}$ experiments, especially with respect to nonlinear experiments for which pulse walk-off management in pump-probe experiments is vital.

This research, initiated during the European contract SPLASH of the 6th European Union (EU) Framework programme, is partly supported by the French ANR project CALIN. S. Mazoyer thanks the Direction Générale de l'Armement (DGA) for financial support and A. Baron acknowledges a fellowship of the Agence Nationale pour le Recherche (ANR).

\section{References}

1. M. Soljacic and J. D. Joannopoulos, "Enhancement of nonlinear effects using photonic crystals," Nat. Mater. 3, 211-219 (2004).

2. M. Notomi, K. Yamada, A. Shinya, J. Takahashi, C. Takahashi, and I. Yokohama, "Extremely Large GroupVelocity dispersion of line-defect waveguides in photonic crystal slabs," Phys. Rev. Lett. 87, 253902 (2001).

3. Y. A. Vlasov, M. O'Boyle, H. F. Hamann, and S. J. McNab, "Active control of slow light on a chip with photonic crystal waveguides," Nature 438, 65-69 (2005).

4. S. Hughes, L. Ramunno, J. F. Young, and J. E. Sipe, "Extrinsic optical scattering loss in photonic crystal waveguides: role of fabrication disorder and photon group velocity," Phys. Rev. Lett. 94, 033903 (2005).

5. E. Kuramochi, M. Notomi, S. Hughes, A. Shinya, T. Watanabe, and L. Ramunno, "Disorder-induced scattering loss of linedefect waveguides in photonic crystal slabs," Phys. Rev. B 72, 161318 (2005).

6. B. Wang, S. Mazoyer, J. P. Hugonin, and P. Lalanne, "Backscattering in monomode periodic waveguides," Phys. Rev. B 78, 245108 (2008).

7. S. Mazoyer, P. Lalanne, J. C. Rodier, J. P. Hugonin, M. Spasenović, L. Kuipers, D. M. Beggs, and T. F. Krauss, "Statistical fluctuations of transmission in slow light photoniccrystal waveguides," Opt. Express 18, 14654-14663 (2010).
8. Y. A. Vlasov, M. A. Kaliteevski, and V. V. Nikolaev, "Different regimes of light localization in disordered photonic crystals," Phys. Rev. B 60, 1555-62 (1999).

9. N. Le Thomas, H. Zhang, J. Jágerská, V. Zabelin, R. Houdré, I. Sagnes, and A. Talneau, "Light transport regimes in slow light photonic crystal waveguides," Phys. Rev. B 80, 125332 (2009).

10. K. A. Atlasov, M. Felici, K. F. Karlsson, P. Gallo, A. Rudra, B. Dwir, and E. Kapon, "1D photonic band formation and photon localization in finite-size photonic-crystal waveguides," Opt. Express 18, 117-122 (2009).

11. P. D. García, S. Smolka, S. Stobbe, and P. Lodahl, "Density of states controls Anderson localization in disordered photonic crystal waveguides," Phys. Rev. B 82, 165103 (2010).

12. J. Topolancik, B. Illic, and F. Vollmer, "Experimental observation of strong photon localization in disordered photonic crystal waveguides," Phys. Rev. Lett. 99, 253901 (2007).

13. A. Melloni, A. Canciamilla, C. Ferrari, F. Morichetti, L. O'Faolain, T. F. Krauss, R. De La Rue, A. Samarelli, and M. Sorel, "Tunable delay lines in silicon photonics: coupled resonators and photonic crystals, a comparison," IEEE Photon. J. 2, 181-194 (2010).

14. C. Monat, B. Corcoran, M. Ebnali-Heidari, C. Grillet, B. J. Eggleton, T. P. White, L. O'Faolain, and T. F. Krauss, "Slow light enhancement of nonlinear effects in silicon engineered photonic crystal waveguides," Opt. Express 17, 2944-53 (2009).

15. A. Baron, A. Ryasnyanskiy, N. Dubreuil, P. Delaye, Q. Vy Tran, S. Combrié, A. de Rossi, R. Frey, and G. Roosen, "Light localization induced enhancement of third order nonlinearities in a GaAs photonic crystal waveguide," Opt. Express 17, 552-557 (2009).

16. K. Suzuki and T. Baba, "Nonlinear light propagation in chalcogenide photonic crystal slow light waveguides," Opt. Express 18, 26675 (2010).

17. S. Mazoyer, J. P. Hugonin, and P. Lalanne, "Disorder-induced multiple scattering in photonic-crystal waveguides," Phys. Rev. Lett. 103, 063903 (2009).

18. G. Lecamp, J. P. Hugonin, and P. Lalanne, "Theoretical and computational concepts for periodic optical waveguides," Opt. Express 15, 11042-60 (2007).

19. L. O'Faolain, S. A. Schulz, D. M. Beggs, T. P. White, M. Spasenovic, L. Kuipers, F. Morichetti, A. Melloni, S. Mazoyer, J. P. Hugonin, P. Lalanne, and T. F. Krauss, "Loss engineered slow light waveguides," Opt. Express 18, 27627-38 (2010)

20. L. A. Coldren and S. W. Corzine, Diode Lasers and Photonic Integrated Circuits (Wiley, 1995).

21. A. Baron, S. Mazoyer, W. Smiga, and P. Lalanne, "Attenuation coefficient of single-mode periodic waveguides," Phys. Rev. Lett. 107, 153901 (2011).

22. B. A. van Tiggelen, A. Lagendijk, M. P. van Albada, and A. Tip, "Speed of light in random media," Phys. Rev. B 45, 12233-12243 (1992).

23. R. T. Chiao and A. M. Steinberg, in Progress in Optics, E. Wolf, ed. (Elsevier, 1997), Vol. XXXVII, p. 345.f. 